\title{
Effect of sub-inhibitory dose of cefotaxime on multidrug resistant
}

\section{Staphylococcus haemolyticus isolates}

\author{
Madhurima Chakraborty $^{a}$, Taniya Bardhana, Manjari Basu ${ }^{b}$, Bornali Bhattacharjee ${ }^{a}$ \\ ${ }^{a}$ National Institute of Biomedical Genomics, Kalyani, West Bengal, India \\ ${ }^{\mathrm{b}}$ College of Medicine \& Jawaharlal Nehru Memorial Hospital, Kalyani, West Bengal, India
}

\section{Corresponding author:}

Bornali Bhattacharjee,

National Institute of Biomedical Genomics, Kalyani, West Bengal, India

E-mail: bornali_27@yahoo.co.uk, bb2@nibmg.ac.in.

\author{
\#91-9830948523
}

\begin{abstract}
Critical care of neonates involves substantial usage of antibiotics and exposure to multi-drug resistant (MDR) nosocomial pathogens. These pathogens are often exposed to sub-MIC doses of antibiotics which might result in a range of physiological effects. There-fore, to understand the outcome of sub-inhibitory dosage of antibiotics on Staphylococcus populations, nasal swab specimens were collected from 34 neonates admitted to the Sick Newborn Care Unit between 2017-2018, a total of 41 non-repetitive isolates were included in this study. Staphylococcus haemolyticus was the prevalent species $(58.54 \%)$ with high nonsusceptibility to cefotaxime (CTX) $(79.16 \%)$, gentamicin $(87.50 \%)$, and meropenem $(54.17 \%)$. Biofilm forming abilities of S. haemolyticus isolates in the presence of sub-optimal CTX $(30 \mu \mathrm{g} / \mathrm{mL})$, the predominantly prescribed $\beta$-lactam antibiotic, were then determined by crystal violet assays and extracellular DNA (eDNA) quantitation. CTX was found to significantly enhance biofilm production among the non-susceptible isolates ( $\mathrm{p}$-value Wilcoxin $_{\text {in }}$ test- 0.000008) with increase in eDNA levels (p-value wilcoxin test- 0.000004). Additionally, no changes in non-susceptibility were observed among populations of two MDR isolates, JNM56C1 and JNM60C2 after $>500$ generations of growth in the absence of antibiotic selection in vitro. These findings demonstrate that sub-MIC concentration of CTX induces biofilm formation and short-term non-exposure to antibiotics does not alter non-susceptibility among $S$. haemolyticus isolates.
\end{abstract}

Keywords: Cefotaxime, S. haemolyticus, neonates, sub-MIC, biofilms, short-term evolution 


\section{Introduction}

Across the world, approximately 2.4 million children lose lives in the first month of birth each year and India contributes majorly to it. India has a neonate mortality rate of $21.7 \%$ [1] and $20-41 \%$ of preterm neonates admitted to tertiary care hospitals succumb to sepsis [2] often ascribed to multidrug resistant (MDR) bacteria [3]. Among the causal pathogens, members of the Enterobacteriaceae and Moraxellaceae families dominate, inspite of that, Coagulase-negative staphylococci (CoNS) have been found to contribute significantly $[4,5]$.

When bacterial populations are exposed to antibiotics, resistance often emerges quickly due to mutations or horizontal gene transfer [6]. Additionally, CoNS are also known for causing device-related infections by forming biofilms [7]. Biofilms, in general, have been reported to have considerably higher resistance to antimicrobial agents as compared to planktonic cells [8] and several studies have found that sub-optimal doses of antibiotics can induce biofilm development in a wide range of species $[9,10,11]$.

The Sick Newborn Care Units (SNCU) are known for considerable use of antibiotics especially third generation cephalosporins, aminoglycosides and penems to a certain extent [12]. This is one of the contributing factors resulting in selection of resistant nosocomial bacteria [13]. In addition, empiric use of the same antibiotics against non-susceptible pathogens among neonates requiring longer duration of stay due to prematurity, might play a vital role in enhancing biofilm-formation. Further, it is also unclear as to how many generations would it take after discontinuation of an antibiotic for a resistant bacterial population to become susceptible again [14].

To address each of these questions, we focused on colonization. Nasally colonizing Staphylococcus spp. among the admitted neonates were characterized to identify the predominant nosocomial multidrug resistant species. The next set of experiments were carried out to understand if sub-optimal dosage of the most prescribed group of antibiotics, 
namely, cefotaxime, a third-generation cephalosporin, results in enhanced biofilm formation in the laboratory. Finally, short-term 15-day evolution experiments were carried out to evaluate if discontinuation of the antibiotic could result in loss of non-susceptibility.

\section{Results}

2.1. Species identification and susceptibility profiling

Among the 34 neonates, the average number of days spent in the SNCU was 7.1. Respiratory distress after birth was the most common clinical symptom $(\mathrm{n}=17 ; 50 \%)$ in the cohort and only 3 preterm along with 4 term born neonates had higher than normal levels of C-Reactive Protein (Supplementary table 1). To characterize the distribution of Staphylococcus spp. colonizing the nares, nasal swabs were collected and spread on MSA upon enrichment. A total of 41 Staphylococcus isolates were characterized (MZ636452MZ636490) from 34 neonates. Staphylococcus haemolyticus was found to be the prevalent species $(\mathrm{n}=24,58.54 \%)$ followed by Staphylococcus aureus $(\mathrm{n}=7,17.07 \%)$ and Staphylococcus epidermidis ( $\mathrm{n}=6,14.63 \%)$ (Figure 1A, Supplementary table 2).

Among the 4 antibiotics tested, maximum number of $S$. haemolyticus isolates were nonsusceptible (Intermediate and Resistant) to GEN ( $\mathrm{n}=21,87.50 \%$; MIC: 6- >256 $\mu \mathrm{g} / \mathrm{mL}$ ), however, $19(79.16 \%)$ were non-susceptible to CTX with MIC values of $>256 \mu \mathrm{g} / \mathrm{mL}$ followed by MRP ( $\mathrm{n}=13,54.17 \%$; MIC: $6-32 \mu \mathrm{g} / \mathrm{mL})$. Only a total of 7 isolates $(29.17 \%)$ were non-susceptible to AK. Out of 5 CTX sensitive isolates all were susceptible to AK and MRP and 3 were susceptible to GEN (Figure 1B, Supplementary table 2). All the other Staphylococcus spp. isolates $(\mathrm{n}=17)$ were susceptible to AK and MRP, 5 were nonsusceptible to GEN and 3 to CTX. $13(54.17 \%)$ of S. haemolyticus isolates harbored both the $m e c A$ and $b l a Z$ genes and another $8(33.33 \%)$ had only the mecA gene. Two CTX susceptible 
isolates with MIC of $8 \mu \mathrm{g} / \mathrm{mL}$ (JNM17C1 and JNM51C1) were also found to harbor the resistance genes (Figure 1C).
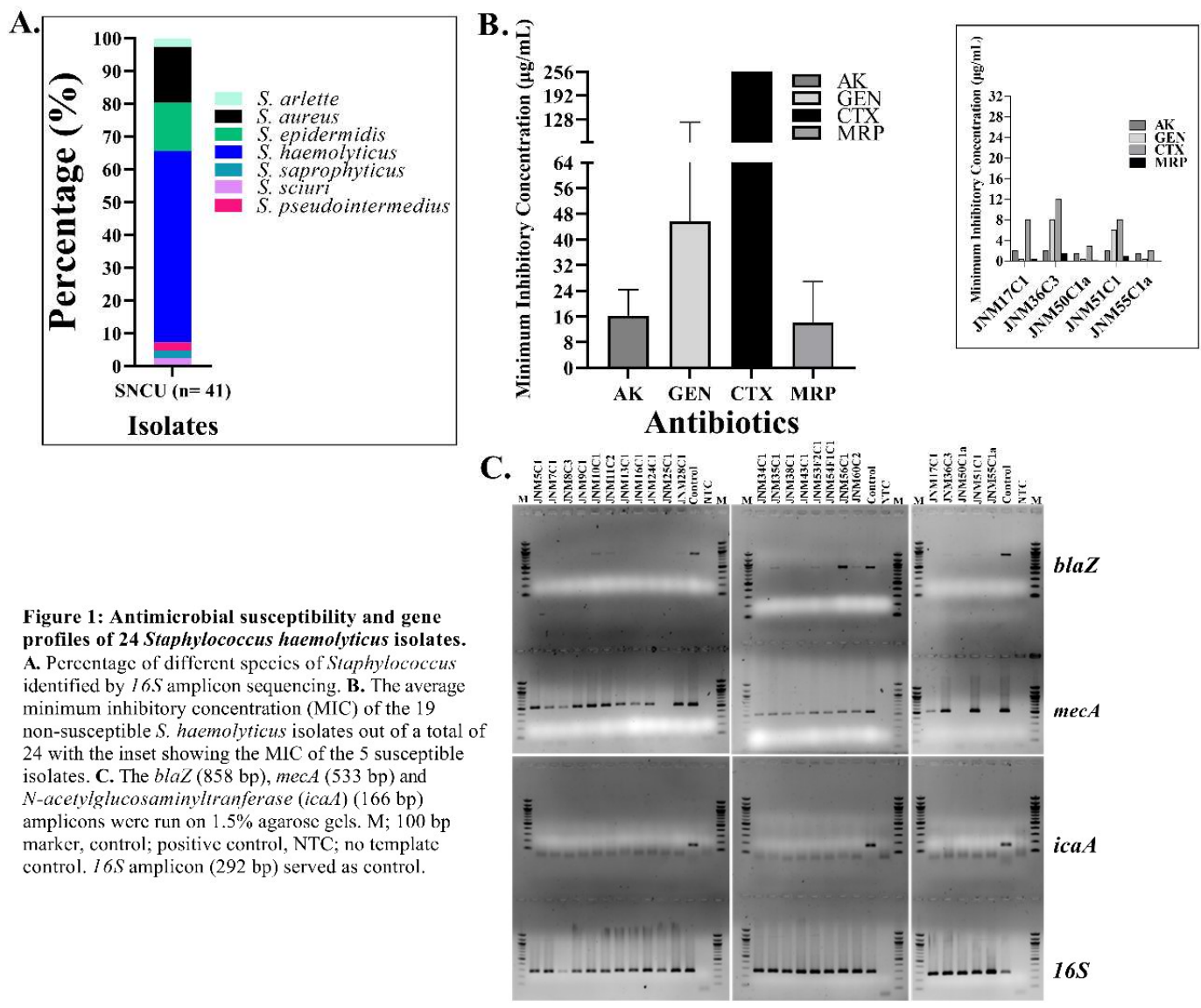

2.2. Biofilm enhancement and eDNA release among $S$. haemolyticus isolates

All except for one $S$. haemolyticus isolate (JNM50C1a), grown in $\mathrm{TSB}_{\mathrm{glu}}$ and $\mathrm{TSB}_{\mathrm{NaCl}}$ were identified to be biofilm producers after 24 hours. The growth media strongly influenced biofilm formation and a total of 14 out of $24(58.33 \%)$ isolates produced biofilms in TSB $_{\text {glu }}$ whereas, $22(91.67 \%)$ isolates formed biofilms in $\mathrm{TSB}_{\mathrm{NaCl}}$. In the presence of sub-inhibitory concentration of CTX $(30 \mu \mathrm{g} / \mathrm{mL})$, biofilm production was enhanced significantly (pvalue Wilcoxin test- 0.000008 ) among all the non-susceptible isolates compared to the controls. By contrast, there was reduction in median optical density values among the susceptible isolates presumably due to cell death (Figure 2A, Supplementary table 3). 
Given that eDNA release has been implicated in S. haemolyticus biofilm formation, whether increased biofilm formation also resulted in an increase in quantities of eDNA in the

A.

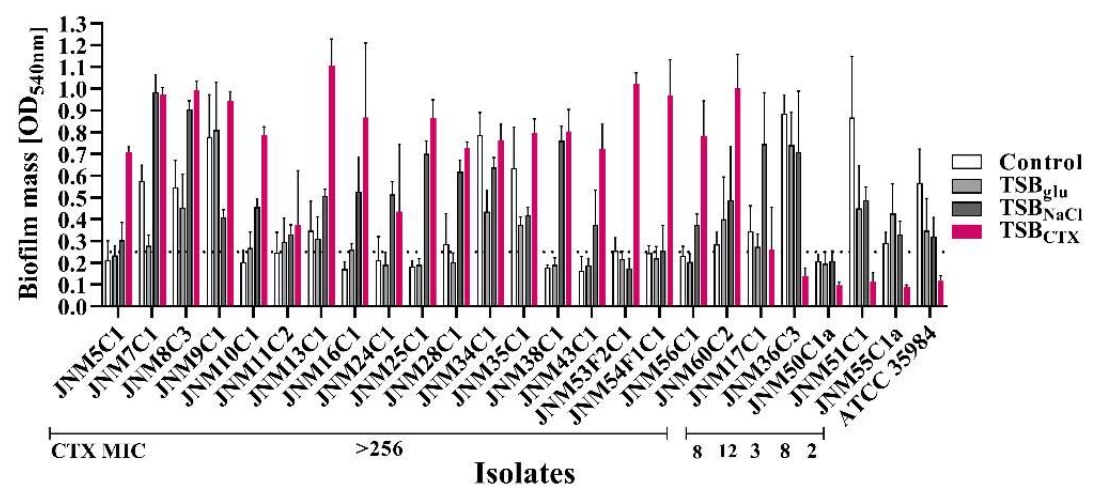

B.

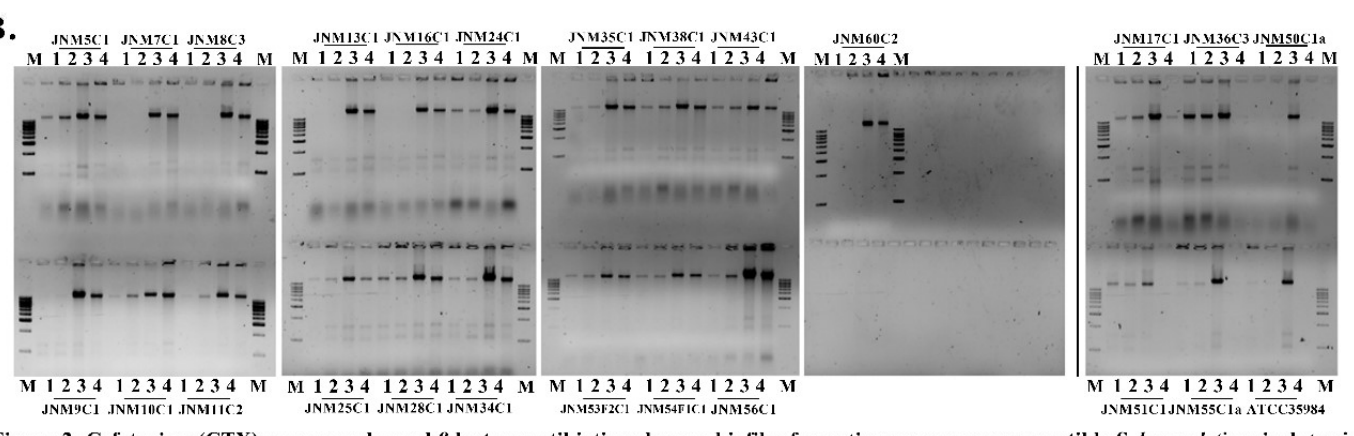

Figure 2: Cefotaxime (CTX), a commonly used $\beta$-lactam antibiotic enhances biofilm formation among non-susceptible $S$. haemolyticus isolates in vitro. A. Semi-quantitative determination of 24 hour biofilm formation under known inducible conditions [tryptic soy broth with $1 \%$ glucose $\left(\mathrm{TSB}_{\mathrm{glu}}\right.$ ) and $3 \%$ sodium chloride $\left.\left(\mathrm{TSB}_{\mathrm{NaC}}\right)\right]$ and $30 \mu \mathrm{g} / \mathrm{mL}$ CTX $\left(\mathrm{TSB}_{\mathrm{CTX}}\right)$ using crystal violet assays. The dotted line denotes the cut-off optical density value for biolilm formation (0.25). The biofilm-forming ATCC 35984 S. epidermidis strain served as a control. B. Biofilm supernatants run on $0.8 \%$ agarose gels to determine the presence of extracellular DNA (eDNA). M; 1000 bp marker.

biofilms was next evaluated. Biofilm forming sessile cells were harvested and removed by centrifugation and the cell-free nucleic acid in the supernatant was harvested and resolved on $0.8 \%$ agarose gels (Figure 2B). Significant increase in eDNA levels were observed in the $\mathrm{TSB}_{\mathrm{NaCl}}\left(\mathrm{p}\right.$-value $\mathrm{Wilcoxin}_{\text {test }}{ }^{-0.000004)}$ and $\mathrm{TSB}_{\mathrm{CTX}}(\mathrm{p}$-value Wilcoxin test- 0.000004$)$ treated groups (Supplementary table 4).

2.3. Whole genome sequencing and resistome mapping of ancestral populations

To generate the resistome profiles of two MDR S. haemolyticus ancestral populations (JNM56C1 and JNM60C2), both non-susceptible to CTX, GEN and MRP, paired-end whole genome sequencing was carried out. Iterative de novo and reference guided assembly (NC_007168) resulted in alignment of $>93 \%$ of error-corrected reads. JNM56C1 was 
determined to be a multi locus sequence type (ST) 38 with a chromosome length of 25,54,979 bp (CP063753) at an average sequencing depth of 310. A total of 6 antimicrobial resistance genes were annotated namely, $A A C\left(6^{\prime}\right)-I e-A P H\left(2^{\prime \prime}\right)-I a, b l a Z, d f r G$, mecA, msrA and mphC. Similarly, JNM60C2, a ST-1 isolate, had a chromosome sequence length of $25,11,057$ (CP065356) which was ascertained at an average depth of 421. The antimicrobial genes identified were $A P H\left(3^{\prime}\right)-I I I a, A A C\left(6^{\prime}\right)-I e-A P H\left(2^{\prime \prime}\right)-I a, b l a Z, d f r G$, mecA and SAT-4.The polysaccharide intercellular adhesin (PIA) operon was confirmed to be absent from both the genomes. None of the isolates were found to harbor any plasmids.

2.4. No change in susceptibility in the absence of antibiotic selection

It has been shown in $S$. aureus strains using in vitro curing assays that harboring SCCmec imposes a fitness cost [22]. However, if a similar cost is attached with nonsusceptibility in S. haemolyticus, a decay of non-susceptibility in the absence of antibiotic selection needs to be tested. To understand this, JNM56C1 and JNM60C2, two completely sequenced multidrug resistant $S$. haemolyticus populations with biofilm forming ability (Figure 3A) and known generation time of 30 and 40 minutes respectively (Supplementary table 5) were serially passaged in triplicate for $>500$ generations (Supplementary table 6) in the absence of antibiotics (Figure 3B). Susceptibility to both $\beta$-lactam (CTX, MRP) and aminoglycoside (AK, GEN) antibiotics were then compared among the 6 evolved clones and the 2 ancestral populations along with candidate resistance gene amplification. There were no differences in CTX and MRP susceptibility or MIC values among each set of ancestral and evolved populations. Both AK and GEN susceptibilities remained unchanged, however, with 0.25 -fold decrease in $\mathrm{AK}$ MIC values in 2 of the 6 evolved populations (Figure 3C, Supplementary table 6). In all evolved populations, resistance genes blaZ, mec $A$ and $A A C\left(6^{\prime}\right)$ - 
$A P H\left(2^{\prime}\right)$ were retained albeit with differences in band intensities from the ancestral populations (Figure 3D).

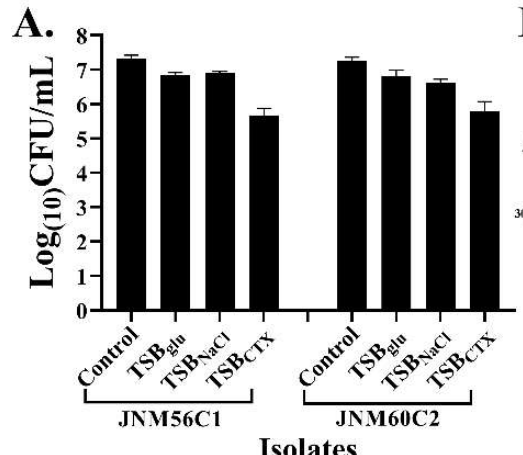

C.

Isolates

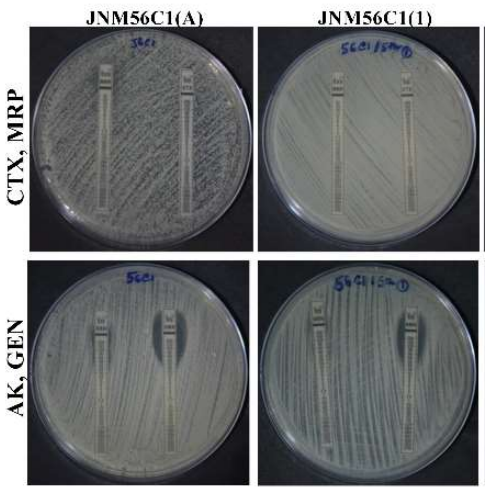

$\mathrm{JNM} 60 \mathrm{C} 2(\mathrm{~A})$
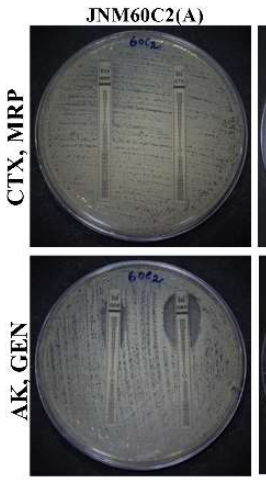

JNM60C2(1)
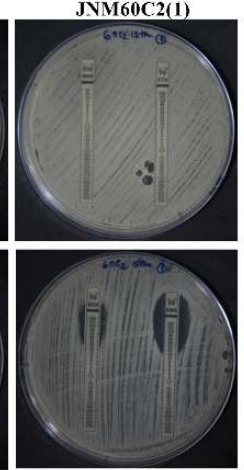

B.

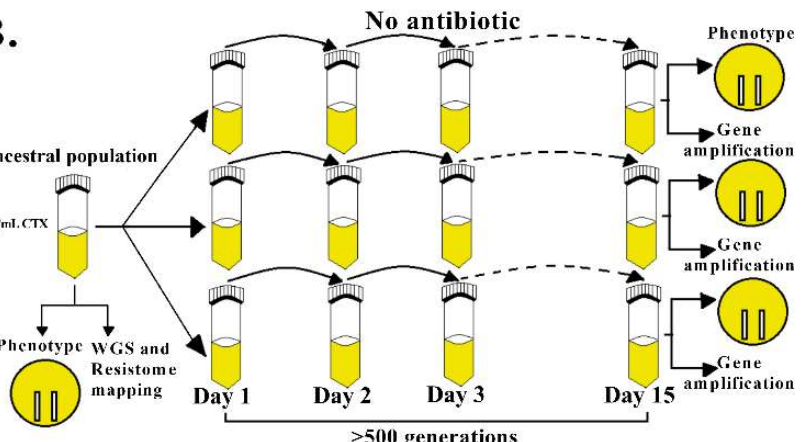

D.

JNM56C1(2)
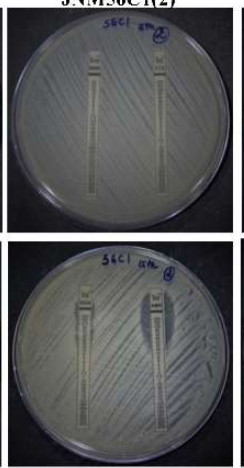

JNM60C2(2)
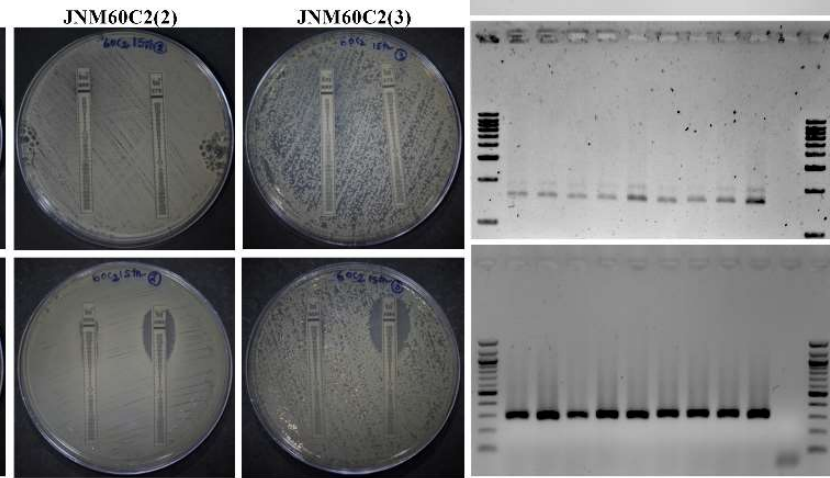

Figure 3: No change in in vitro antibiotic susceptibility among MDR, biofilm forming S. haemolyticus isolates (JNM56C1 and JNM60C2) grown in the absence of antibiotic in short-term evolution experiments. A. The presence of living sessile cells is confirmed by $\log _{(10)} \mathrm{CFU}$ counts in both the isolates in the presence of $30 \mu \mathrm{g} / \mathrm{mL}$ CTX. The error bars represent standard deviation across three technical replicates. B. Study design of the evolution experiment. C.

Comparison of susceptibility to $\beta$-lactam and aminoglycoside antibiotics among ancestral and evolved populations using E-tests. D.The blaZ (858 bp, panel 1), mecA (533 bp, panel 2) and $A A C\left(6^{\prime}\right)-A P I I\left(2^{\prime}\right)$ (1658 bp, panel 3 ) and $16 S(292 \mathrm{bp}$, panel 4) genes were amplified from the ancestral and evolved populations and amplicons were run on $0.8-1.5 \%$ agarose gels. M; 100/1000 bp marker, control; postive control, NTC; no template control. 


\section{Discussion}

Increase in the emergence of MDR pathogens is the major cause of mortality in neonatal sepsis due to the ineffectiveness of first line of antibiotics recommended by the World Health Organization [23]. Neonates admitted to the SNCU, or Newborn Intensive Care Units (NICU) are prone to anticipatory broad spectrum antibiotic therapies [24], possess an immature immune system [25] and have been observed to be colonized by pathogens $[26,27]$ which make them extremely susceptible to infections. Hence, this study was undertaken to understand the role of antibiotic use and disuse on multi-resistant Staphylococcus spp. that frequently colonize neonate nares in an SNCU with high usage of $\beta$-lactam antibiotics. MDR S. haemolyticus, an emerging pathogen known for multi-resistance $[28,29]$ was observed to be the most frequent colonizer with $79.16 \%$ non-susceptibility to CTX and $>256 \mu \mathrm{g} / \mathrm{mL}$ MIC value in each case.

To understand if an in vitro equivalent of the therapeutic dose of CTX $(30 \mu \mathrm{g} / \mathrm{mL})[18]$ could affect the physiology of high MIC resistant colonizers, we chose to delineate biofilm formation which is known to accentuate antibiotic resistance and colonization. $S$. haemolyticus lacking the PIA operon has been shown to form biofilms in vitro under different growth conditions $[17,30,31,32]$ and the same were used to identify the biofilm-formers. A total of 23 out of 24 isolates were identified to be biofilm formers inspite of the absence of PIA. However, we found $\mathrm{TSB}_{\mathrm{Nacl}}$ to be a better media for in vitro production compared to $\mathrm{TSB}_{\mathrm{glu}}$, in contrast to the previous studies $[17,31]$. Escalation of biofilm formation in the presence of insufficient antibiotics has been observed in a variety of species through a variety of mechanisms $[9,10,11]$, inspite of that, not much work has been done to understand biofilm development in S. haemolyticus and there exists only a single report where nosocomial clones were found to show enhanced biofilm formation on glass and polystyrene surfaces in the presence of $1 / 4$ MIC of three antibiotics namely, oxacillin, vancomycin, and linezolid 
[32].Therefore, this study is one of the first showing increase in biofilm formation and eDNA release among MDR, nosocomial S. haemolyticus isolates in the presence of sub-inhibitory concentration of CTX.

Evolved antibiotic resistance is a costly affair for many species of bacteria and often result in decreased competitive fitness in the absence of selection pressure [33]. Nevertheless, studies have also exhibited that often bacteria can tackle such trade-offs by way of mutations that compensate the changes [34] and in a long-term evolution experiment comprising of an ancestral streptomycin resistant Escherichia coli population by Lamrabet et al, it was clearly demonstrated that after 50, 000 generations of growth in the absence of antibiotic there was no change in susceptibility [35]. Similarly, in our short-term evolution experiments $(>500$ generations) we observed no changes in the MIC values of CTX and MRP in the evolved clones as compared to both the ancestral populations and minor changes in 1 clone per population in case AK and GEN. However, we believe that a better approach would have been whole genome sequencing of the evolved clones instead of a candidate gene approach to gauge the loss of resistance genes if any in these experiments.

\section{Materials and Methods}

\subsection{Sample collection and isolation of bacterial colonies}

Nasal swab samples were collected from 34 neonates (Term born= 7, moderately preterm $=11$ and, very or extremely preterm=16) admitted after birth to the SNCU of College of Medicine \& JNM Hospital, Kalyani between 2017-2018 (Supplementary table 1). This study was approved by the Hospital and Institutional Ethical Committees. Consenting mothers signed a consent form prior to sample collection. Specimens were grown in Muller Hinton broth (MHB) (Himedia labs, Mumbai, India) and plated on Mannitol salt agar (MSA) (Himedia labs), a selective agar and grown aerobically at $37^{\circ} \mathrm{C}$. Single colonies were 
collected and subcultured. Two colonies per sample were isolated from MSA plates (Himedia labs) except for one specimen (JNM8) and two neonates who were followed up on day 4 and 3 respectively (JNM53 and JNM54). The colonies were subcultured in MHB (Himedia labs) and $20 \%$ DMSO (Himedia labs) stocks were maintained at $-80^{\circ} \mathrm{C}$ for further analyses.

\subsection{Bacterial DNA isolation and species identification}

Genomic DNA was isolated from 2 subcultured isolates per specimen using the QIAamp DNA extraction mini kit (Qiagen, Hilden, Germany). Amplification and Sanger sequencing of $16 \mathrm{SrRNAgene}$ were carried out using the S-D-Bact-0008-c-S-20/ S-D-Bact-1391-a-A-17 primer pairs [15] (Eurofins Scientific, Bengaluru, India) to identify the species. One colony per subject was included in the study unless different species were identified.

\subsection{Minimum Inhibitory Concentration (MIC) determination}

The minimum inhibitory concentration (MIC) values were determined using MIC strips (Himedia Labs). The antibiotics tested were cefotaxime (CTX), aminoglycosides; amikacin (AK) and gentamicin (GEN) and the carbapenem; meropenem (MRP). Experiments were carried out according to Clinical and Laboratory Standards Institute (CLSI) guidelines [16].

\subsection{Quantification of biofilms}

The biofilm forming ability of Staphylococcus isolates was determined by a modified crystal violet assay method as described previously [17]. Briefly, 96well polystyrene, flatbottom microtiter plates were filled with $180 \mu \mathrm{L}$ of tryptic soy broth (TSB) (Himedia labs) and $20 \mu \mathrm{L}$ bacterial cells grown to a Macfarland score of 0.5 in brain heart infusion broth (BHI) (Himedia labs) were added and incubated at $37^{\circ} \mathrm{C}$ for 24 hours (hrs) statically. Biofilm forming capacities of all isolates were determined in TSB, TSB with $1 \%$ glucose (TSB glu $_{\text {), }}$ 
TSB with $3 \% \mathrm{NaCl}\left(\mathrm{TSB}_{\mathrm{NaCl}}\right.$ ), and TSB with $30 \mu \mathrm{g} / \mathrm{mL}$ CTX (TSB $\mathrm{CTX}$ ) which is an in vitro equivalent of the therapeutic dosage [18]. After $24 \mathrm{hrs,} \mathrm{planktonic} \mathrm{cells} \mathrm{were} \mathrm{removed,}$ adherent cells were fixed with 99\% methanol (Finar chemicals, Ahmedabad, India) for 10 mins, and plates were washed once with $1 \times$ PBS (Sigma-Aldrich,St. Louis, United States) and air-dried for 10 mins. Modified crystal violet assays were performed, and the absorbance (OD) was recorded at $540 \mathrm{~nm}$. The assays were performed with 6 replicates for each condition in 2 parallel runs. The isolates with an OD of $\geq 0.25$ were considered biofilm positive. S. epidermidis ATCC 35984 was used as a positive control. The presence of living sessile cells was determined by colony-forming units (CFU) for selected isolates in the presence of $\mathrm{TSB}_{\mathrm{glu}}, \mathrm{TSB}_{\mathrm{NaCl}}$ and $\mathrm{TSB}_{\mathrm{CTX}}$ in triplicate.

\subsection{Extracellular DNA (eDNA) quantification}

Extracellular DNA was extracted as previously described by Kaplan et al. with modification [9]. Biofilms were grown in triplicate in TSB, TSB ${ }_{\text {glu, }}, \mathrm{TSB}_{\mathrm{NaCl}}$ and $\mathrm{TSB}_{\mathrm{CTX}}$ in 24 well polystyrene microtiter plates, in a total volume of $1 \mathrm{~mL}$ per well. After $24 \mathrm{hrs}$ of growth, the liquid was carefully removed, the plates were washed once with 1XPBS (SigmaAldrich, United States) and $50 \mu 1$ of Tris-EDTA buffer (10 mM Tris-HCl, $1 \mathrm{mM}$ EDTA, pH 7.4) (Sigma-Aldrich, United States) was added to each well. The biofilm-forming cells were scraped off the bottom surface of the wells and were transferred to $1.5 \mathrm{~mL}$ microcentrifuge tubes. The tubes were centrifuged at $13,000 \mathrm{rpm}$ for $25 \mathrm{~s}$ and $8 \mu 1$ of each of the supernatants were resolved on $0.8 \%$ agarose gels. Densitometric analyses of eDNA were carried out using Image Lab software version 6.0.1(Bio-Rad Laboratories, Hercules, United States). 
4.6. Genomic DNA isolation and whole-genome sequencing

Two isolates (JNM56C1 and JNM60C2) were subcultured in the presence of $30 \mu \mathrm{g} / \mathrm{mL}$ CTX. Total DNA from both were purified using the QIAamp DNA extraction mini kit (Qiagen, Germany) and were subjected to paired end whole genome sequencing $(2 \times 300 \mathrm{bp})$ on an Illumina HiSeq2500 platform (Illumina, San Diego, USA). Both de novo and reference guided assembly was carried out using Velvet and Bowtie2 respectively $[19,20]$ to build genomes as described previously [21].

\subsection{Short-term evolution experiment}

The isolates grown in the presence of $30 \mu \mathrm{g} / \mathrm{mL}$ CTX formed the ancestral population for each. Generation time for both were calculated from growth curves. The two populations were serially passaged for $>500$ generations in triplicate for 15 days in fresh Luria-Bertani (LB) media (Himedia labs) in the absence of antibiotic selection at a dilution of 1:100. The ancestral and evolved populations were tested for antibiotic susceptibility for $\beta$-lactam (CTX, MRP) and aminoglycoside (AK, GEN) antibiotics by Etests.

\subsection{Amplification of genes}

The presence of $\beta$-lactamase genes mecA and blaZ, aminoglycoside resistance gene $A A C\left(6^{\prime}\right)-A P H\left(2^{\prime}\right)$, and $\mathrm{N}$-acetylglucosaminyltransferase icaA gene were detected by using PCR amplification. The positive controls used were a laboratory isolate for the $\beta$-lactamase and aminoglycoside resistance gene and S. epidermidis ATCC 35984 for icaA gene amplification. A 292 basepair (bp) region of the $16 S$ gene was amplified as an internal control. Supplementary table 7 lists all the primers used in this study. 


\subsection{Statistical analyses}

Normality across datasets was evaluated using the Kolmogorov-Smirnov test. To identify significant differences among experimental conditions tested, the Wilcoxon test was performed using GraphPad Prism version 9.1.2 (GraphPad Software, La Jolla, California, USA). A p-value of $<0.05$ was considered to be statistically significant.

\section{Conclusions}

To summarize, this study highlights the high nasal carriage rates of MDR $S$. haemolyticus isolates among neonates admitted to the SNCU, demonstrates that these isolates have a tendency towards enhanced biofilm formation upon exposure to insufficient quantities of CTX and further reveals no change in susceptibility to both commonly used $\beta$-lactams and aminoglycosides when grown in the absence of selection pressure in the short-term. All these findings further reinforce the idea that a definite means of reducing antibiotic resistance is antibiotic stewardship.

Author Contributions: MC ran all the laboratory experiments, analyzed the data and wrote the first draft of the manuscript. TB ran the initial screening experiments and standardized the biofilm assays. MB was the clinician who was involved in specimen collection. BB conceptualized all the experiments, carried out all the next generation sequencing analyses, collated all the data and wrote and reviewed the final draft.

Funding: This work was supported by the Ramanujan fellowship (SB/S2/RJN-013/2014) funded by Science and Engineering Research Board, Department of Science and Technology, Government of India and intramural funding awarded to B.B. 
Institutional Review Board Statement: The study was conducted according to the guidelines of the Declaration of Helsinki and approved by the College of Medicine \& Jawaharlal Nehru Memorial Hospital and National Institute of Biomedical Genomics Ethics Committees. The hospital ethical committee reference number is F24/Pr/COMJNMH/IEC/16/536.

Informed Consent Statement: Informed consent was obtained from all the parents of the subjects involved in the study.

Data Availability Statement: The annotated genome sequences (CP063753, CP065356) and the $16 S$ amplicon sequences (MZ636452-MZ636490) are available at the GenBank database.

Acknowledgments: The authors acknowledge Dr. Samsiddhi Bhattacharjee, NIBMG, Kalyani, India for providing valuable inputs on statistical analyses.

Conflicts of Interest: None

\section{References}

1. Neonatal mortality-UNICEF Data: https://data.unicef.org/topic/child-survival/neonatalmortality/, 2020

2. Jain, K.; Sankar, M.J.; Nangia, S.; Ballambattu, V.B.; Sundaram, V.; Ramji, S; Plakkal, N.; Kumar, P.; Jain, A.; Sivanandan, S.; et al. Causes of death in preterm neonates ( $<33$ weeks) born in tertiary care hospitals in India: analysis of three large prospective multicentric cohorts. J. Perinatol. 2019, 39,13-19. doi: 10.1038/s41372-019-0471-1.

3. Wattal, C.; Kler, N.; Oberoi, J.K.; Fursule, A.; Kumar, A.; Thakur. A. Neonatal Sepsis: Mortality and Morbidity in Neonatal Sepsis due to Multidrug-Resistant (MDR) Organisms: Part 1. Indian J. Pediatr. 2020, 87,117-121. doi: 10.1007/s12098-019-03106-z.

4. Investigators of the Delhi Neonatal Infection Study (DeNIS) collaboration. Characterisation and antimicrobial resistance of sepsis pathogens in neonates born in tertiary care centres in Delhi, India: a cohort study. Lancet Glob. Health 2016, 4, 752-60. doi: 10.1016/S2214-109X(16)30148-6. 
5. Nazir, A. Neonatal sepsis due to coagulase negative Staphylococci: a study from Kashmir valley, India. Int. J. Contemp. Pediatr. 2019, 6,650-5. DOI: http://dx.doi.org/10.18203/23493291.ijcp20190705.

6. Blair, J.M.; Webber, M.A.; Baylay, A.J.; Ogbolu, D.O.; Piddock, L.J. Molecular mechanisms of antibiotic resistance. Nat. Rev. Microbiol. 2015, 13,42-51. doi:

10.1038/nrmicro3380.

7. Mack, D.; Rohde, H.; Harris, L.G.; Davies, A.P.; Horstkotte, M.A.; Knobloch, J.K. Biofilm formation in medical device-related infection. Int. J. Artif. Organs 2006, 29,343-59. doi: $10.1177 / 039139880602900404$.

8. Bardhan, T.; Chakraborty, M.; Bhattacharjee, B. Bactericidal Activity of Lactic Acid against Clinical, Carbapenem-Hydrolyzing, Multi-Drug-Resistant Klebsiella pneumoniae Planktonic and Biofilm-Forming Cells. Antibiotics 2019, 8,181. https://doi.org/10.3390/antibiotics8040181.

9. Kaplan, J.B.; Izano, E.A.; Gopal, P.; Karwacki, M.T.; Kim, S.; Bose, J.L.; Bayles, K.W.; Horswill, A.R. Low levels of $\beta$-lactam antibiotics induce extracellular DNA release and biofilm formation in Staphylococcus aureus. mBio 2012,3,00198-12. doi: 10.1128/mBio.00198-12.

10. Yu, W.; Hallinen, K.M.; Wood, K.B. Interplay between Antibiotic Efficacy and DrugInduced Lysis Underlies Enhanced Biofilm Formation at Subinhibitory Drug Concentrations. Antimicrob. Agents Chemother. 2017,62,01603-17. doi: 10.1128/AAC.01603-17.

11. Hoffman, L.R.; D'Argenio, D.A.; MacCoss, M.J.; Zhang, Z.; Jones, R.A; Miller, S.I. Aminoglycoside antibiotics induce bacterial biofilm formation. Nature 2005, 436,1171-5. doi: 10.1038/nature03912.

12. Ramasamy, S.; Biswal, N.; Bethou, A.; Mathai, B. Comparison of two empiric antibiotic regimen in late onset neonatal sepsis--a randomized controlled trial. J. Trop. Pediatr. 2014,60,83-6. doi: 10.1093/tropej/fmt080.

13. Ramasethu, J. Prevention and treatment of neonatal nosocomial infections. Matern. Health Neonatol. Perinatol. 2017, 3, 5. doi: 10.1186/s40748-017-0043-3.

14. Yen, P.; Papin, J.A. History of antibiotic adaptation influences microbial evolutionary dynamics during subsequent treatment. PLoS Biol. 2017, 15, 2001586. doi: 10.1371/journal.pbio.2001586.

15. Bardhan, T.; Chakraborty, M.; Bhattacharjee, B.; Prevalence of Colistin-Resistant, Carbapenem-Hydrolyzing Proteobacteria in Hospital Water Bodies and Out-Falls of West Bengal, India. International Journal of Environmental Research and Public Health 2020, 17, 1007. https://doi.org/10.3390/ijerph17031007.

16. Clinical and Laboratory Standards Institute. Performance Standards for Antimicrobial Susceptibility Testing; CLSI M100-S27.Clinical and Laboratory Standards Institute: Wayne, PA, USA, 2017.

17. Fredheim, E.G.; Klingenberg, C.; Rohde, H.; Frankenberger, S.; Gaustad, P.; Flaegstad, T.; Sollid, J.E. Biofilm formation by Staphylococcus haemolyticus. J. Clin. Microbiol. 2009; 47, 1172-80. doi: 10.1128/JCM.01891-08.

18. Brochmann, P.R.; Hesketh, A.; Jana, B.; Brodersen, G.H.; Guardabassi, L. Transcriptome analysis of extended-spectrum $\beta$-lactamase-producing Escherichia coli and methicillin- 
resistant Staphylococcus aureus exposed to cefotaxime. Sci. Rep. 2018, 8, 16076. doi: 10.1038/s41598-018-34191-3.

19. Zerbino, D.R.; Birney, E. Velvet: algorithms for de novo short read assembly using de Bruijn graphs. Genome Res. 2008, 18, 821-9. doi: 10.1101/gr.074492.107.

20. Langmead, B.; Salzberg, S.L. Fast gapped-read alignment with Bowtie 2. Nat. Methods 2012, 9, 357-9. doi: 10.1038/nmeth.1923.

21. Bhattacharjee, B.; Bardhan, T.; Chakraborty, M.; Basu, M. Resistance profiles and resistome mapping of multidrug resistant carbapenem-hydrolyzing Klebsiella pneumoniae strains isolated from the nares of preterm neonates. Int. J. Antimicrob. Agents 2019, 53, 535 537. doi: 10.1016/j.ijantimicag.2018.12.002.

22. Ender, M.; McCallum, N.; Adhikari, R.; Berger-Bächi, B. Fitness cost of SCCmec and methicillin resistance levels in Staphylococcus aureus. Antimicrob. Agents Chemother. 2004, 48, 2295-7. doi: 10.1128/AAC.48.6.2295-2297.2004.

23. Chaurasia, S.; Sivanandan, S.; Agarwal, R.; Ellis, S.; Sharland, M.; Sankar, M.J.

Neonatal sepsis in South Asia: huge burden and spiralling antimicrobial resistance. BMJ 2019 , 364, k5314. doi: 10.1136/bmj.k5314.

24. Patel, S.J.; Oshodi, A.; Prasad, P.; Delamora, P.; Larson, E.; Zaoutis, T.; Paul, D.A.; Saiman, L. Antibiotic use in neonatal intensive care units and adherence with Centers for Disease Control and Prevention 12 Step Campaign to Prevent Antimicrobial Resistance.

Pediatr. Infect. Dis. J. 2009, 28, 1047-51. doi: 10.1097/INF.0b013e3181b12484. 
25. Simon, A.K.; Hollander, G.A.; McMichael, A. Evolution of the immune system in humans from infancy to old age. Proc. Biol. Sci. 2015,282, 20143085. doi: 10.1098/rspb.2014.3085.

26. Dias, M.; Saleem, J. Surface colonization and subsequent development of infections with multi drug resistant organisms in a neonatal intensive care unit. Ann. Clin. Microbiol. Antimicrob. 2019, 18, 12. doi: 10.1186/s12941-019-0312-2.

27. Labi, A.K.; Bjerrum, S.; Enweronu-Laryea, C.C.; Ayibor, P.K.; Nielsen, K.L.; Marvig, R.L.; Newman, M.J.; Andersen, L.P.; Kurtzhals, J.A.L. High Carriage Rates of Multidrug-Resistant GramNegative Bacteria in Neonatal Intensive Care Units From Ghana. Open Forum Infect. Dis. 2020, 7 , ofaa109. doi: 10.1093/ofid/ofaa109.

28. Cavanagh, J.P.; Klingenberg, C.; Hanssen, A.M.; Fredheim, E.A.; Francois, P.; Schrenzel, J.; Flægstad, T.; Sollid, J.E. Core genome conservation of Staphylococcus haemolyticus limits sequence based population structure analysis. J. Microbiol. Methods 2012, 89, 159-66. doi: 10.1016/j.mimet.2012.03.014.

29. Cavanagh, J.P.; Hjerde, E.; Holden, M.T.; Kahlke, T.; Klingenberg, C.; Flægstad, T.; Parkhill, J.; Bentley, S.D.; Sollid, J.U. Whole-genome sequencing reveals clonal expansion of multiresistant Staphylococcus haemolyticus in European hospitals. J. Antimicrob. Chemother. 2014, 69, 2920-7. doi: $10.1093 / \mathrm{jac} / \mathrm{dku} 271$.

30. Panda, S.; Jena, S.; Sharma, S.; Dhawan, B.; Nath, G.; Singh, D.V. Identification of Novel Sequence Types among Staphylococcus haemolyticus Isolated from Variety of Infections in India. PLoS One 2016, 11, e0166193. doi: 10.1371/journal.pone.0166193.

31. Panda, S.; Singh, D.V. Biofilm Formation by ica-Negative Ocular Isolates of Staphylococcus haemolyticus. Front. Microbiol. 2018, 9, 2687. doi: 10.3389/fmicb.2018.02687.

32. Pereira-Ribeiro, P.M.; Sued-Karam, B.R.; Faria, Y.V.; Nogueira, B.A.; Colodette, S.S.; Fracalanzza, S.E.; Duarte, J.L.; Júnior, R.H.; Mattos-Guaraldi, A.L. Influence of antibiotics on biofilm formation by different clones of nosocomial Staphylococcus haemolyticus. Future Microbiol. 2019, 14, 789-799. doi: 10.2217/fmb-2018-0230.

33. Andersson, D.I.; Hughes, D. Antibiotic resistance and its cost: is it possible to reverse resistance? Nat. Rev. Microbiol. 2010, 8, 260-71. doi: 10.1038/nrmicro2319.

34. Nagaev, I.; Björkman, J.; Andersson, D.I.; Hughes, D. Biological cost and compensatory evolution in fusidic acid-resistant Staphylococcus aureus. Mol. Microbiol. 2001, 40, 433-9. doi: 10.1046/j.13652958.2001.02389.x.

35. Lamrabet, O.; Martin, M.; Lenski, R.E.; Schneider, D. Changes in Intrinsic Antibiotic Susceptibility during a Long-Term Evolution Experiment with Escherichia coli. mBio 2019,10,e00189-19. doi: 10.1128/mBio.00189-19. 